\title{
Cusp relation for the Pauli potential
}

\author{
H. Levämäki, ${ }^{1,2}$ Á. Nagy, ${ }^{3}$ K. Kokko, ${ }^{1,2}$ and L. Vitos ${ }^{4,5,6}$ \\ ${ }^{1}$ Department of Physics and Astronomy, University of Turku, FI-20014 Turku, Finland \\ ${ }^{2}$ Turku University Centre for Materials and Surfaces (MatSurf), Turku, Finland \\ ${ }^{3}$ Department of Theoretical Physics, University of Debrecen, H-4010 Debrecen, Hungary \\ ${ }^{4}$ Applied Materials Physics, Department of Materials Science and Engineering, Royal Institute of Technology, Stockholm SE-100 44, Sweden \\ ${ }^{5}$ Institute for Solid State Physics and Optics, Wigner Research Centre for Physics, Hungarian Academy of Sciences, \\ P.O. Box 49, H-1525 Budapest, Hungary \\ ${ }^{6}$ Department of Physics and Astronomy, Division of Materials Theory, Uppsala University, Box 516, SE-75121 Uppsala, Sweden
}

(Received 15 October 2014; published 22 December 2014)

\begin{abstract}
In orbital-free density functional theory, only a Schrödinger-like equation has to be solved for the square root of the electron density. In this equation, however, there is an extra potential in addition to the Kohn-Sham potential, the so-called Pauli potential. Cusp relations are now presented for this Pauli potential for spherically symmetric systems.
\end{abstract}

DOI: 10.1103/PhysRevA.90.062515

PACS number(s): 31.15.E-

\section{INTRODUCTION}

Electron density is the key quantity in the density functional theory. According to the Hohenberg-Kohn theorems [1] the external potential $v(\mathbf{r})$ is determined within a trivial additive constant by the electron density $n(\mathbf{r})$ and there exists a variational principle that leads to the Euler equation:

$$
\frac{\delta E}{\delta n}=\mu \text {. }
$$

As the kinetic energy functional is unknown, one can turn to the Kohn-Sham scheme [2], that is, a noninteracting system. There the electrons move independently in a common, local external potential and the density

$$
n(\mathbf{r})=\sum_{i}^{N}\left|\phi_{i}(\mathbf{r})\right|^{2}
$$

is the same as the true interacting electron density. The orbitals $\phi_{i}$ satisfy the Kohn-Sham equations

$$
\left[-\frac{1}{2} \nabla^{2}+v_{\mathrm{KS}}(\mathbf{r})\right] \phi_{i}(\mathbf{r})=\varepsilon_{i} \phi_{i}(\mathbf{r}),
$$

where $N, \varepsilon_{i}$, and $v_{\mathrm{KS}}$ are the number of electrons, the oneelectron energies, and the Kohn-Sham potential, respectively. (Atomic units are used in the paper.)

The noninteracting kinetic energy

$$
T_{s}=-\frac{1}{2} \sum_{i}^{N} \int \phi_{i}^{*} \nabla^{2} \phi_{i} d \mathbf{r}
$$

can be partitioned as $T_{s}=T_{w}+T_{p}$, where $T_{w}$ is the Weizsäcker kinetic energy [3]

$$
T_{w}=-\frac{1}{2} \int n^{1 / 2} \nabla^{2} n^{1 / 2} d \mathbf{r} .
$$

The Pauli energy is defined as $T_{p}=T_{s}-T_{w}$ [4-19].

The Euler equation (1) can be rewritten as

$$
\left[-\frac{1}{2} \nabla^{2}+v_{p}+v_{\mathrm{KS}}\right] n^{1 / 2}=\mu n^{1 / 2},
$$

where

$$
v_{p}=\frac{\delta T_{p}}{\delta n}
$$

is the Pauli potential, the functional derivative of the Pauli energy $T_{p}$.

Recently, there has been a growing interest in the orbitalfree density functional theory. It has the great advantage that only one equation, the Euler equation [Eq. (1) or (6)] should be solved instead of several Kohn-Sham equations. It is very important when the system considered has a lot of electrons. The disadvantage is the lack of knowledge of the exact form of the kinetic energy (or the Pauli energy) functional. There exist several approximations [20-33] that can be applied in orbital-free calculations. Recently a novel approach [34-36] that avoids using approximate kinetic energy functionals has been proposed for spherically symmetric systems.

The Pauli potential has a very important role in orbitalfree density functional theory. Therefore it is essential to know its properties. Exact relations are valuable from several points of view, e.g., helpful in constructing approximate functionals. Several fundamental characteristics have already been presented [7,37-41]. In this paper, the cusp relation is explored. It is very useful in numerical calculations. The present study is restricted to spherically symmetric densities. The cusp condition is derived in the following section. The last section presents some numerical demonstration.

\section{CUSP RELATION FOR THE PAULI POTENTIAL}

We derived ground- and excited-state cusp conditions for the electron density [42-44] that can be utilized in the derivation. Further important results on cusp relations for the density can be found in [45-52]. Consider the Kohn-Sham potential

$$
\begin{gathered}
v_{\mathrm{KS}}=-\frac{Z}{r}+w, \\
w=v_{J}+v_{\mathrm{xc}},
\end{gathered}
$$

where $Z, v_{J}$, and $v_{\mathrm{xc}}$ are the atomic number and the Coulomb and exchange-correlation potentials, respectively. The expansion of the potential $w$ around $r=0$ leads to [42-44,50,52]

$$
w=A+B r+C r^{2}+\cdots,
$$


where $A, B, C, \ldots$ are constants. The solution of the KohnSham equations [2] can then be written as

$$
\phi_{n}=\sum_{l m} r^{l}\left(c_{n l m}^{(0)}+c_{n l m}^{(1)} r+c_{n l m}^{(2)} r^{2}+\cdots\right) Y_{l m}(\Omega) \text {. }
$$

Here $Y_{l m}$ are the spherical harmonics. The relationship between the coefficients in Eqs. (10) and (11) can be written as [42-44]

$$
\begin{gathered}
c_{n l m}^{(1)}=-\frac{Z}{l+1} c_{n l m}^{(0)}, \\
c_{n l m}^{(2)}=\frac{1}{2 l+3}\left[\frac{Z^{2}}{l+1}-\varepsilon_{n}+A\right] c_{n l m}^{(0)},
\end{gathered}
$$

and

$$
\begin{aligned}
c_{n l m}^{(3)}= & -\frac{Z}{3(l+1)(l+2)}\left\{(3 l+4) c_{n l m}^{(2)}\right. \\
& \left.-\left[\frac{Z}{l+1}+(l+1) B\right] c_{n l m}^{(0)}\right\} .
\end{aligned}
$$

Then the density has the form $[44,52]$

$$
\begin{aligned}
n(r)= & n(0)\left[(1-Z r)^{2}+\frac{1}{3}\left[Z^{3}+w^{\prime}(0)\right] r^{3}\right] \\
& +\zeta\left(r^{2}-\frac{5}{3} Z r^{3}\right)+\xi\left(r^{2}-Z r^{3}\right)+o\left(r^{4}\right),
\end{aligned}
$$

where

$$
\begin{gathered}
w^{\prime}(0)=B \\
\zeta=\frac{1}{2 \pi} \sum_{n} c_{n 00}^{(0)} c_{n 00}^{(2)},
\end{gathered}
$$

and

$$
\xi=\frac{1}{4 \pi} \sum_{n} \sum_{m=-1}^{m=1}\left(c_{n 1 m}^{(0)}\right)^{2} .
$$

From Eq. (6) it follows that

$$
v_{p}=\mu-v_{\mathrm{KS}}+\frac{1}{2} \frac{\nabla^{2} n^{1 / 2}}{n^{1 / 2}} .
$$

It can also be written as

$$
v_{p}=\mu-v_{\mathrm{KS}}+\frac{1}{4} \frac{\nabla^{2} n}{n}-\frac{1}{8}\left(\frac{\nabla n}{n}\right)^{2} .
$$

Applying the density (15), we arrive at

$$
\begin{aligned}
\frac{1}{2} \frac{\nabla^{2} n^{1 / 2}}{n^{1 / 2}}= & -\frac{Z}{r}-Z^{2}+\frac{3}{2} \frac{\zeta+\xi}{n(0)} \\
& +\left(\frac{2 Z \xi}{n(0)}+w^{\prime}(0)\right) r+\cdots
\end{aligned}
$$

for the last term of Eq. (19). Therefore, the expansion of the Pauli potential takes the form

$$
v_{p}=\mu-Z^{2}-w(0)+\frac{3}{2} \frac{\zeta+\xi}{n(0)}+\frac{2 Z \xi}{n(0)} r+\cdots .
$$

It means that the Pauli potential at the nucleus is

$$
v_{p}(0)=\mu-Z^{2}-w(0)+\frac{3}{2} \frac{\zeta+\xi}{n(0)},
$$

while its derivative is

$$
v_{p}^{\prime}(0)=\frac{2 Z \xi}{n(0)} .
$$

One can immediately notice that the quantity $\xi$ [Eq. (18)] can be expressed with the spherically averaged $p$-electron density $\bar{n}_{p}$

$$
\xi=\left.\frac{\bar{n}_{p}}{r^{2}}\right|_{r=0}
$$

Consequently,

$$
v_{p}^{\prime}(0)=\left.\frac{2 Z}{n(0)} \frac{\bar{n}_{p}}{r^{2}}\right|_{r=0} .
$$

These expressions can be transformed into more useful relations if we express $\xi$ and $\zeta$ with the density and its derivatives at the nucleus. From Eq. (15) we immediately obtain that

$$
n^{\prime \prime}(0)=2\left[Z^{2} n(0)+\zeta+\xi\right]
$$

and

$$
n^{\prime \prime \prime}(0)=2 n(0)\left[Z^{3}+w^{\prime}(0)\right]-10 Z \zeta-6 Z \xi .
$$

Therefore

$$
v_{p}(0)=\mu-\frac{5}{2} Z^{2}-w(0)+\frac{3}{4} \frac{n^{\prime \prime}(0)}{n(0)},
$$

and

$$
v_{p}^{\prime}(0)=-6 Z^{3}-w^{\prime}(0)+\frac{5 Z n^{\prime \prime}(0)+n^{\prime \prime \prime}(0)}{2 n(0)} .
$$

Note that we can really obtain the sum of the potentials $v_{p}$ and $w$ from Eqs. (20), (29), and (30). However, while the Pauli potential is completely of kinetic origin, the potential $w$ is the sum of the classical Coulomb and the exchange-correlation potentials. Thus, $w$ is calculated and/or approximated independently of $v_{p}$. Moreover, there exist cusp relations for $w$ [42-44,50,52]. Therefore, Eqs. (29) and (30) can be useful in checking the accuracy of orbital-free calculations.

\section{ILLUSTRATIVE EXAMPLES AND DISCUSSION}

To illustrate the results presented in the previous section we performed calculations for the $\mathrm{Be}, \mathrm{Ne}, \mathrm{Ar}, \mathrm{Kr}$, and $\mathrm{Xe}$ atoms. A recently published robust and general solver for the radial Kohn-Sham equations [53] was applied. Figure 1 presents the Pauli potential and its radial derivative for the Be and Xe atoms. The plot was obtained from converged densities and Kohn-Sham potentials by inverting the Euler equation (6) for $v_{p}(r)$. The Pauli potential ensures that the Pauli principle is fulfilled. If bosons were treated instead of fermions, all particles would occupy the lowest energy state (in the ground state) and no extra term would be added to the one-particle potential in the Schrödinger-like equation for the square root of the density. In the case of fermions, however, the particles fill levels starting with the lowest energy up to $\mu$ and the Pauli potential is different from zero if the system has more than two electrons. It is well known that the radial density reflects the shell structure. The shell structure can be clearly seen in 

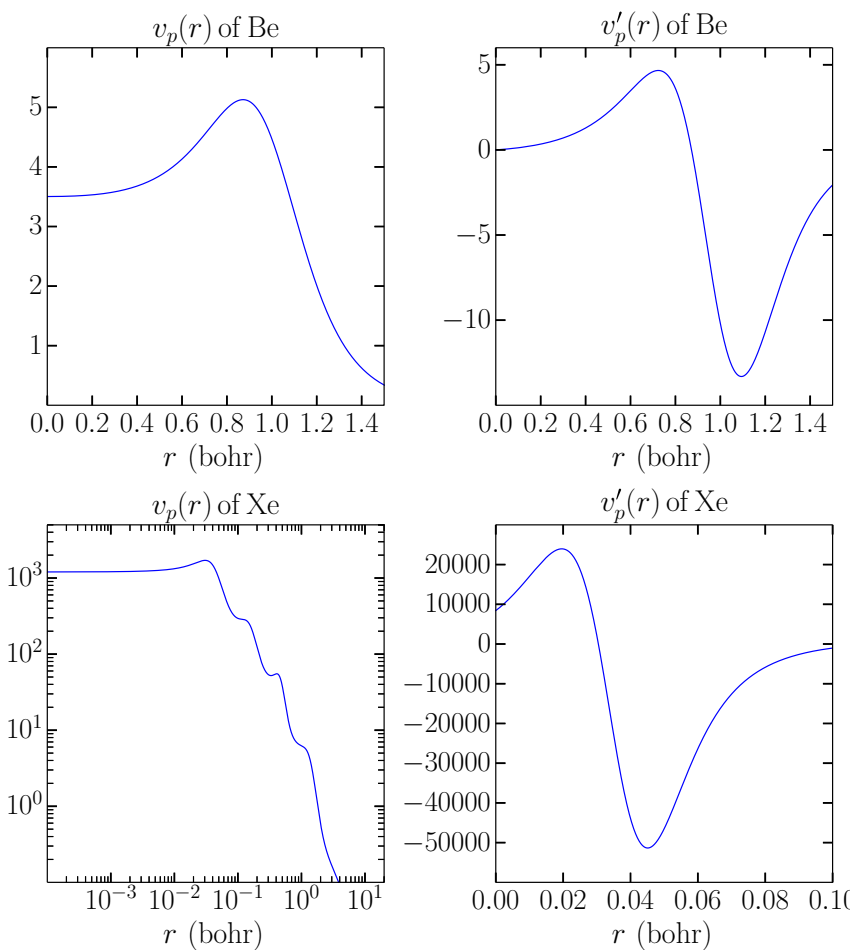

FIG. 1. (Color online) Pauli potentials $v_{p}(r)$ and their radial derivatives $v_{p}^{\prime}(r)$ (in atomic units) for $\mathrm{Be}$ and $\mathrm{Xe}$ atoms near the nucleus. They have been obtained from converged densities and Kohn-Sham potentials by inverting the Euler equation (6) for $v_{p}(r)$, that is, using Eq. (19). Values near the nucleus are seen to agree with those [Eqs. (29) and (30)] given in Table I.

the Pauli potential, too (Fig. 1). This behavior of the Pauli potential was demonstrated earlier in [11].

Table I presents the values of $v_{p}(r)$ and $v_{p}^{\prime}(r)$ at the nucleus for $\mathrm{Be}, \mathrm{Ne}, \mathrm{Ar}, \mathrm{Kr}$, and $\mathrm{Xe}$ atoms. Equations (29) and (30) were applied using self-consistently determined densities and Kohn-Sham potentials. For comparison, $v_{p}(r)$ and $v_{p}^{\prime}(r)$ are
TABLE I. Numerically determined values of $v_{p}(r)$ and $v_{p}^{\prime}(r)$ at the nucleus for $\mathrm{Be}, \mathrm{Ne}, \mathrm{Ar}, \mathrm{Kr}$, and $\mathrm{Xe}$ atoms (in atomic units) supplying Eqs. (29) and (30) and applying Eq. (19).

\begin{tabular}{lccccc}
\hline \hline & $\mathrm{Be}$ & $\mathrm{Ne}$ & $\mathrm{Ar}$ & $\mathrm{Kr}$ & $\mathrm{Xe}$ \\
\hline$v_{p}(0)$ [Eqs. (29)-(30)] & 3.5 & 29.2 & 111.8 & 505.5 & 1201 \\
$v_{p}(0)$ [Eq. (19)] & 3.5 & 29.2 & 111.9 & 505.5 & 1202 \\
$v_{p}^{\prime}(0)$ [Eqs. (29)-(30)] & 0.0 & 12.9 & 165.0 & 2079 & 8422 \\
$v_{p}^{\prime}(0)$ [Eq. (19)] & 0.0 & 12.8 & 172.0 & 2064 & 8415 \\
\hline \hline
\end{tabular}

also determined from Fig. 1 [that is, inverting Eq. (6), which leads to Eq. (19)]. A third-order polynomial was applied to extract these values. There is a good agreement between the values obtained by the two methods.

The cusp relations for the density are well known and can be easily computed, therefore Eqs. (29) and (30) can be easily applied. Note that the the sums $v_{p}(0)+w(0)$ and $v_{p}^{\prime}(0)+$ $w^{\prime}(0)$ can be expressed with only the density and its derivatives at the nucleus. It is worth mentioning that $\mu$ determines the large $r$ behavior of the density. That is, both the atomic number and $\mu$ can be obtained from the density.

The results presented in this article might be useful not only in spherically symmetric systems. For example, they can be applied for muffin-tin orbitals of solid-state calculations [54]. In summary, cusp relations are derived for the Pauli potential in spherically symmetric systems. It is shown that the expressions for the sum of Pauli and the classical Coulomb and exchange correlation potentials and its derivative at the nucleus contain only the density and its derivatives at the nucleus.

\section{ACKNOWLEDGMENTS}

This work was supported by the TAMOP 4.2.2.A$11 / 1 /$ KONV-2012-0036 project. The project is cofinanced by the European Union and the European Social Fund. Grants OTKA No. K 100590 and No. 109570 are also gratefully acknowledged.
[1] P. Hohenberg and W. Kohn, Phys. Rev. 136, B864 (1964).

[2] W. Kohn and L. J. Sham, Phys. Rev. 140, A1133 (1965).

[3] C. F. Weizsäcker, Z. Phys. 96, 431 (1935).

[4] M. Levy, J. P. Perdew, and V. Sahni, Phys. Rev. A 30, 2745 (1984).

[5] N. H. March, Phys. Lett. A 113, 476 (1986).

[6] N. H. March, J. Comput. Chem. 8, 375 (1987).

[7] M. Levy and Hui Ou-Yang, Phys. Rev. A 38, 625 (1988).

[8] C. Herring and M. Chopra, Phys. Rev. A 37, 31 (1988).

[9] A. Holas and N. H. March, Phys. Rev. A 44, 5521 (1991).

[10] Á. Nagy and N. H. March, Phys. Chem. Liq. 22, 129 (1990).

[11] Á. Nagy, Acta Phys. Hung. 70, 321 (1991).

[12] Á. Nagy and N. H. March, Int. J. Quantum Chem. 39, 615 (1991).

[13] Á. Nagy and N. H. March, Phys. Chem. Liq. 25, 37 (1992).

[14] Á. Nagy and N. H. March, Phys. Chem. Liq. 38, 759 (2000).

[15] N. H. March and Á. Nagy, Phys. Rev. A 78, 044501 (2008).
[16] N. H. March and Á. Nagy, Phys. Rev. A 81, 014502 (2010).

[17] N. H. March, Á. Nagy, F. Bogár, and F. Bartha, Phys. Chem. Liq. 50, 412 (2012).

[18] N. H. March, J. Mol. Struct. 943, 77 (2010).

[19] V. G. Tsirelson, A. I. Stash, V. V. Karasiev, and S. Liu, Comp. Theor. Chem. 1006, 92 (2013).

[20] E. Chacón, J. E. Alvarellos, and P. Tarazona, Phys. Rev. B 32, 7868 (1985).

[21] P. García-González, J. E. Alvarellos, and E. Chacón, Phys. Rev. B 53, 9509 (1996).

[22] P. García-González, J. E. Alvarellos, and E. Chacón, Phys. Rev. B 57, 4857 (1998).

[23] Y. A. Wang and E. A Carter, in Theoretical Methods in Condensed Phase Chemistry, Progress in Theoretical Chemistry and Physics, Book 5, edited by S. D. Schwartz (Kluwer, Dordrecht, 2000), p. 117.

[24] V. L. Ligneres and E. Carter, in Handbook of Materials Modeling, edited by S. Yip (Springer, Dordrecht, 2005), p. 137. 
[25] H. Chen and A. Zhou, Numer. Math. Theor. Methods Appl. 1, 1 (2008).

[26] C. Huang and E. A. Carter, Phys. Rev. B 81, 045206 (2010).

[27] V. V. Karasiev, R. S. Jones, S. B. Trickey, and F. E. Harris, in New Developments in Quantum Chemistry, edited by L. Paz and A. J. Hernandez (Research Signposts, Thiruvananthapuram, Kerala, India, 2009), p. 25.

[28] T. A. Wesolowski, J. Phys. Chem. 97, 8050 (1993).

[29] K. M. Carling and E. A. Carter, Modelling Simul. Mater. Sci. Eng. 11, 339 (2003).

[30] I. Shin and E. A. Carter, Model. Simulat. Mater. Sci. Eng. 20, 015006 (2012).

[31] L. Hung and E. A. Carter, Model. Simulat. Mater. Sci. Eng. 19, 045002 (2011).

[32] S. B. Trickey, V. V. Karasiev, and A. Vela, Phys. Rev. B 84, 075146 (2011).

[33] I. Shin and E. A. Carter, J. Chem. Phys. 140, 18A531 (2014).

[34] Á. Nagy, J. Chem. Phys. 135, 044106 (2011).

[35] Á. Nagy, Int. J. Quantum Chem. 110, 2117 (2010).

[36] Á. Nagy, Chem. Phys. Lett. 460, 343 (2008).

[37] V. V. Karasiev, R. S. Jones, S. B. Trickey, and F. E. Harris, Phys. Rev. B 80, 245120 (2009).

[38] V. V. Karasiev and S. B. Trickey, Comput. Phys. Commun. B 183, 2519 (2012).
[39] S. B. Trickey, V. V. Karasiev, and R. S. Jones, Int. J. Quantum Chem. 109, 2943 (2009).

[40] R. A. King and N. C. Handy, Phys. Chem. Chem. Phys. 2, 5049 (2000).

[41] S. B. Liu and P. W. Ayers, Phys. Rev. A 70, 022501 (2004).

[42] Á. Nagy and K. D. Sen, J. Phys. B 33, 1745 (2000).

[43] Á. Nagy and K. D. Sen, Chem. Phys. Lett. 332, 154 (2000).

[44] Á. Nagy and K. D. Sen, J. Chem. Phys. 115, 6300 (2001).

[45] T. Kato, Commun. Pure Appl. Math. 10, 151 (1957).

[46] E. Steiner, J. Chem. Phys. 39, 2365 (1963); N. H. March, SelfConsistent Fields in Atoms (Pergamon, Oxford, 1975).

[47] N. C. Handy, in Quantum Mechanical Simulation Methods for Studying Biological Systems, edited by D. Bicout and M. Field (Springer-Verlag, Heidelberg, 1996), p. 1.

[48] C. C. J. Roothaan and P. S. Kelly, Phys. Rev. 131, 1177 (1963).

[49] R. T. Pack and W. B. Brown, J. Chem. Phys. 45, 556 (1966).

[50] R. O. Esquivel, J. Chen, M. J. Stott, R. P. Sagar, and V. H. Smith Jr., Phys. Rev. A 47, 936 (1993).

[51] R. O. Esquivel, R. P. Sagar, V. H. Smith, Jr., J. Chen, and M. J. Stott, Phys. Rev. A 47, 4735 (1993).

[52] Z. Qian, Phys. Rev. B 75, 193104 (2007).

[53] O. Certik, J. E. Pask, and J. Vackár, Comp. Phys. Commun 184, 1777 (2013).

[54] L. Vitos, Computational Quantum Mechanics for Materials Engineers: The EMTO Method and Applications (Springer, Berlin, 2007). 